\title{
Current Issues in Kaon Photoelectro-Production off the Nucleon
}

\section{Patrick ACHENBACH*}

Institut für Kernphysik, J. J. Becherweg 45, Johannes Gutenberg-Universität, D-55099 Mainz, Germany

E-mail: patrick@kph.uni-mainz.de

The electromagnetic kaon production amplitudes associated to $\Lambda / \Sigma^{0}$ hyperons can be described by phenomenological models, most notably by isobar approaches.

Experimental data on kaon production have been collected at ELSA, SPring8, GRAAL, LNS Tohoku, and Jefferson Lab in the past, the measurements at Jefferson Lab providing the largest kinematic coverage and statistical significance. However, ambiguities inherent in the models, some data inconsistency in the cross-sections taken at different laboratories, and the problem of missing acceptance in forward direction of the experimental set-ups hinders a reliable extraction of resonance parameters.

Predictions for the hypernuclear photo-production cross-section rely on a consistent and comprehensive description of the elementary process at forward kaon angles, where the current strong variation of the models is very unsatisfactory.

A number of new experiments are now addressing these issues, among them the charged kaon electro-production programme with the KAOS spectrometer at the Mainz Microtron MAMI. In this work predictions of the two prominent isobar models, Kaon-Maid and Saclay-Lyon A, are compared for the kinematics at MAMI.

PACS: 13.60.Le, 25.20.Lj, 25.30.Rw, 21.80.+a

XLVIII International Winter Meeting on Nuclear Physics in Memoriam of Ileana Iori 25-29 January 2010

Bormio, Italy

\footnotetext{
* Speaker.
} 


\section{Introduction}

The kaon electro-production reaction on a proton target is a promising channel for studying the excited nucleon. During the last decade, key kaon production measurements were performed at ELSA, the Electron Stretcher Accelerator in Bonn, published by the SAPHIR collaboration $[1,2]$, and at Jefferson Lab (Thomas Jefferson National Accelerator Facility). Experiment E98-108 in Hall A took data in a kinematic region of high squared-momentum-transfer $Q^{2}=2.35(\mathrm{GeV} / c)^{2}$ and hadronic energy $W \geq 1.80$ [3]. Several large-acceptance measurements have been performed with the CLAS detector at Hall B $[4,5]$. New measurements at CLAS have been performed with improved statistical significance and wider energy coverage compared to previous results [6]. However, the detector CLAS has a limited acceptance for four-vector momentum transfers $Q^{2}<0.5(\mathrm{GeV} / c)^{2}$ and for forward angles. Experiment E03-018 carried out at Hall C studied the two hyperon channels $\mathrm{p}\left(e, e^{\prime} K^{+}\right) \Lambda$ and $\mathrm{p}\left(e, e^{\prime} K^{+}\right) \Sigma^{0}$ and performed the first precise Rosenbluth separation of cross-sections into longitudinal and transverse terms [7, 8]. In spite of all these data, cross-sections published after 1990 and taken at different laboratories are inconsistent and most of the experimental set-ups used to study the strangeness production channels are missing acceptance in forward acceptance.

Although it is believed that the Standard Model provides the right theoretical basis for the description of the process, the non-perturbative nature of QCD at low energies precludes a direct comparison of experiments and theory. In analogy to the successful description of pion photoproduction in the $\Delta$-resonance region or $\eta$ photoproduction in the second resonance region, the electromagnetic kaon production amplitudes associated to $\Lambda / \Sigma^{0}$ hyperons can be described by phenomenological models. Theoretical groups have developed a particular type of effective Lagrangian model, commonly referred to as isobar approach, in which the reaction amplitude is constructed from $s^{-}, t-$, or $u$-channel exchange diagrams. Most of the models use single-channel approaches, in which a single hadron is exchanged. Since several resonances may contribute in this channel, models disagree on their relative importance, and many free parameters have to be fixed.

There are still a number of open problems in the interpretation of kaon photoelectro-production data and the description of the process using phenomenological models, see Ref. [9] for a recent discussion. Especially the shape discrepancy at $W \approx 1.9 \mathrm{GeV}$ is problematic, and partial-wave analyses in this energy region have produced various resonance contributions, including $D_{13}, P_{13}$, $D_{11}$, and $S_{11}$ states.

To conclude, it is fair to say that new experimental data on strangeness production will challenge and improve our understanding of the strong interaction in the low energy regime of QCD.

\section{Phenomenological Description of the Reaction}

In isobaric models the reaction amplitudes are constructed from lowest-order (so-called treelevel) Born terms with the addition of extended Born terms for intermediate particles, $N, K$, or $Y$ resonances, exchanged in the $s-, t$-, and $u$-channels as shown in Fig. 1. Each intermediate state enters into the model through its coupling constant and decay width. Diagrams containing intermediate nucleon resonances can produce peaks at given hadronic energies $W$, or equivalently $\sqrt{s}$, 

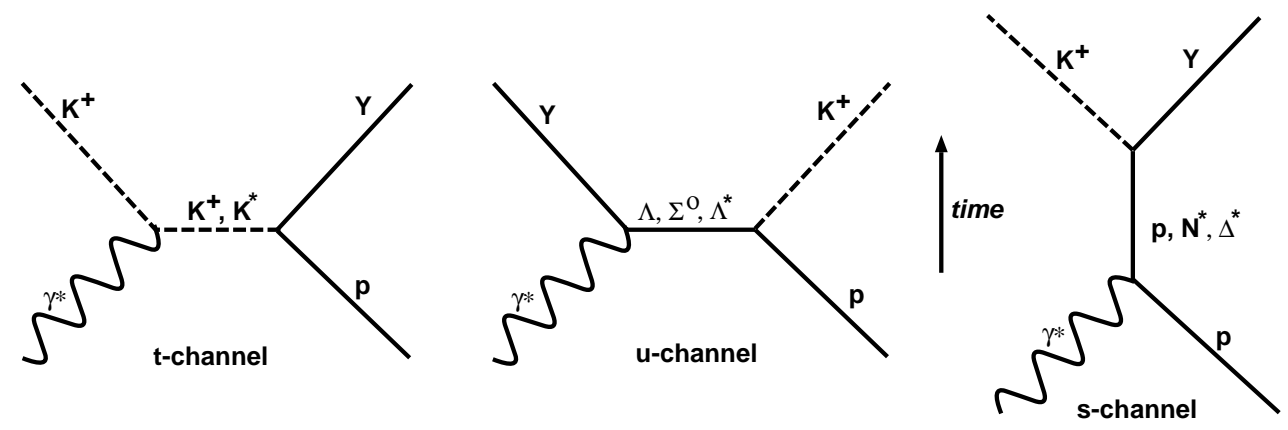

Figure 1: Lowest-order Feynman diagrams of $s^{-}, t$-, and $u$-channel exchanges for the $\mathrm{p}\left(e, e^{\prime} K^{+}\right) Y$ reaction. The vertices enter into the models through coupling constants and form-factors. The different resonance contributions are subject to model variations and global fits.

in the cross-sections at the pole masses of the $s$-channel intermediate states. Intermediate kaons and hyperons cause no such peaking behaviour, and are often called background contributions.

A complete description of the reaction process would require all possible channels that could couple to the initial and final state. Most of the model calculations for kaon electrophoto-production have been performed in the framework of tree-level isobar models [10-12], however, only few coupled-channels calculations exist $[13,14]$. In the tree-level framework higher-order mechanisms like final-state interactions and channel couplings are not treated explicitly.

The advantages of the one-channel, tree-level approach are its limited complexity and the identification of the dominant trends. Even so, several dozen parameters remain. One reason is that, in contrast to pion and eta production, the kaon production process in the $s$-channel is not dominated by a single resonant state. Although the choice of the resonances is guided by existing $\pi N$ data and quark model predictions, the models differ in the use of specific nucleon, hyperon, and kaon resonances. The drawback of the isobaric models is the large and unknown number of exchanged hadrons that can contribute in the intermediate state of the reaction. Depending on which set of resonances is included, very different conclusions about the strengths of the contributing diagrams for resonant baryon formation and kaon exchange may be reached.

For an unpolarised electron beam and an unpolarised target, the five-fold differential crosssection for the $\mathrm{p}\left(e, e^{\prime} K^{+}\right) \Lambda$ process can be written, see $e . g$. [15], in a very intuitive form:

$$
\frac{d \sigma}{d E_{e^{\prime}} d \Omega_{e} d \Omega_{K}^{*}}=\Gamma_{v} \frac{d \sigma_{v}}{d \Omega_{K}^{*}}
$$

where the virtual photo-production cross-section is conventionally expressed as

$$
\frac{d \sigma_{v}}{d \Omega_{K}^{*}}=\frac{d \sigma_{T}}{d \Omega_{K}^{*}}+\varepsilon \frac{d \sigma_{L}}{d \Omega_{K}^{*}}+\sqrt{2 \varepsilon(1+\varepsilon)} \frac{d \sigma_{L T}}{d \Omega_{K}^{*}} \cos \phi_{K}^{*}+\varepsilon \frac{d \sigma_{T T}}{d \Omega_{K}^{*}} \cos 2 \phi_{K}^{*} .
$$

The kaon angles $\theta_{K}^{*}$ and $\phi_{K}^{*}$ are given in spherical coordinates in the hadronic centre-of-mass system. The degree-of-polarisation of the virtual photon is denoted by $\varepsilon$. The terms indexed by $T, L, L T, T T$ are the transverse, longitudinal and interference cross-sections. The electro-production of strangeness introduces two contributions, that are vanishing for the kinematic point at $Q^{2}=0$ : the longitudinal coupling of the photons in the initial state, and the electromagnetic and hadronic 

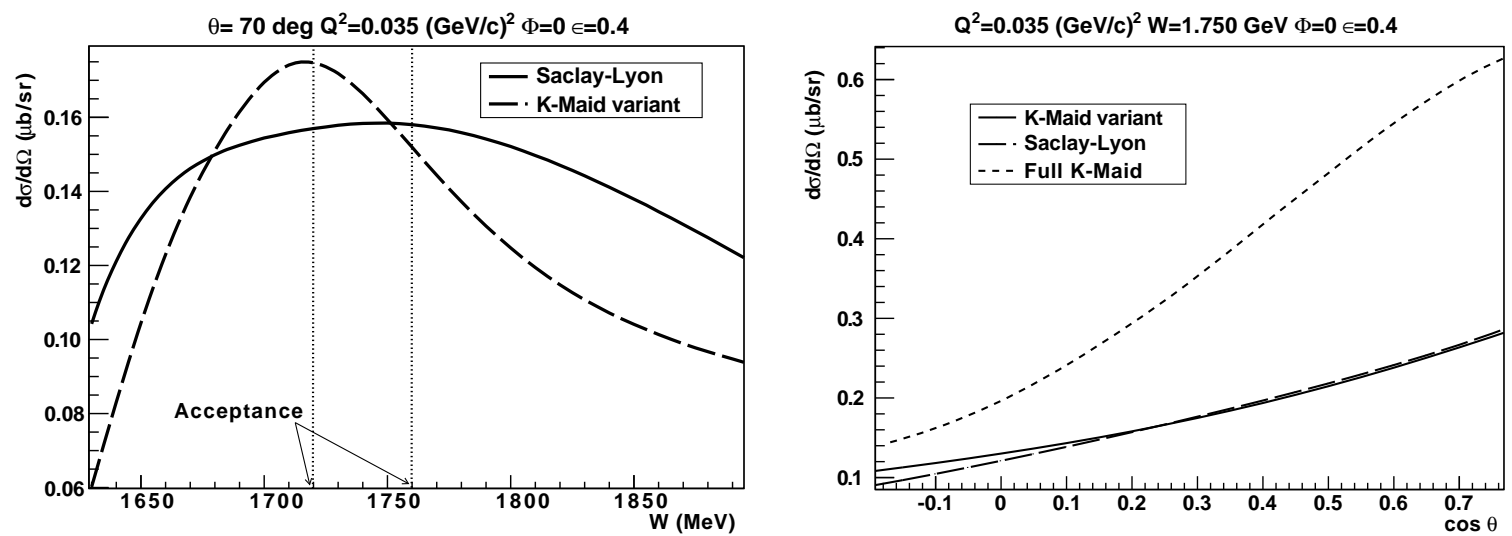

Figure 2: Comparison of cross-section predictions from the Kaon-Maid and Saclay-Lyon models for the kinematics measured at MAMI as a function of the hadronic energy (left) and as a function of the centre-ofmass kaon scattering angle (right). The hadronic energy acceptance of the spectrometer set-up at MAMI for the second kinematic setting is indicated. The acceptance in $\cos \theta$ is between 0.1 and 0.7 .

form factors of the exchanged particles. The introduction of form factors has a serious impact on the properties of a model. It is common practice to use phenomenological form factors to account for the extension of the point-like interactions at the hadronic vertices [16]. It is a well-known phenomenon that the insertion of strong form factors breaks the gauge invariance of isobar models for meson photo- and electro-production. Different models typically have different prescriptions for restoring gauge invariance.

\section{Kaon-Electroproduction at MAMI}

A first experiment of $\Lambda$ and $\Sigma^{0}$ hyperons in elementary electro-production at MAMI was carried out at the spectrometer facility of the A1 Collaboration at the Institut für Kernphysik in Mainz, Germany. During the last years the facility has been extended by the magnetic spectrometer KAOS, dedicated to the detection of charged kaons. The electron beam impinged with an energy of $1507 \mathrm{MeV}$ on a liquid-hydrogen target. The data were taken at two different settings with kaons in a large in-plane angular range, $\vartheta_{K}=21-43^{\circ}$, and in the momentum range of 400$700 \mathrm{MeV} / c$. The two settings were at four-vector momentum transfers of $\left\langle Q^{2}\right\rangle=0.050(\mathrm{GeV} / c)^{2}$, respectively $0.036(\mathrm{GeV} / c)^{2}$, and at hadronic energies of $\langle W\rangle=1.67 \mathrm{GeV}$, respectively $1.75 \mathrm{GeV}$. Cross-sections are to be extracted in the near future. The detection of kaons at very forward laboratory angles will be achieved in the near future by steering the primary beam through the spectrometer and a magnetic chicane comprising two compensating sector magnets.

Model predictions for centre-of-mass cross-sections as a function of the hadronic energy at fixed kaon angle and for the differential cross-section at fixed hadronic energy are shown in Fig. 2. The predictions are from the Kaon-Maid model, a variant of it, and the Saclay Lyon A (SLA) model [17]. Common to the Kaon-Maid and Saclay-Lyon models is that, besides the extended Born diagrams, they also include kaon resonances $K^{*}(890)$ and $K_{1}(1270)$. In Kaon-Maid, four nucleon resonances, the $S_{11}(1650), P_{11}(1710), P_{13}(1720)$, and the "missing resonance" $D_{13}(1900)$ have been included. This $D_{13}$ state has never been observed in pionic reactions but the existence of this 

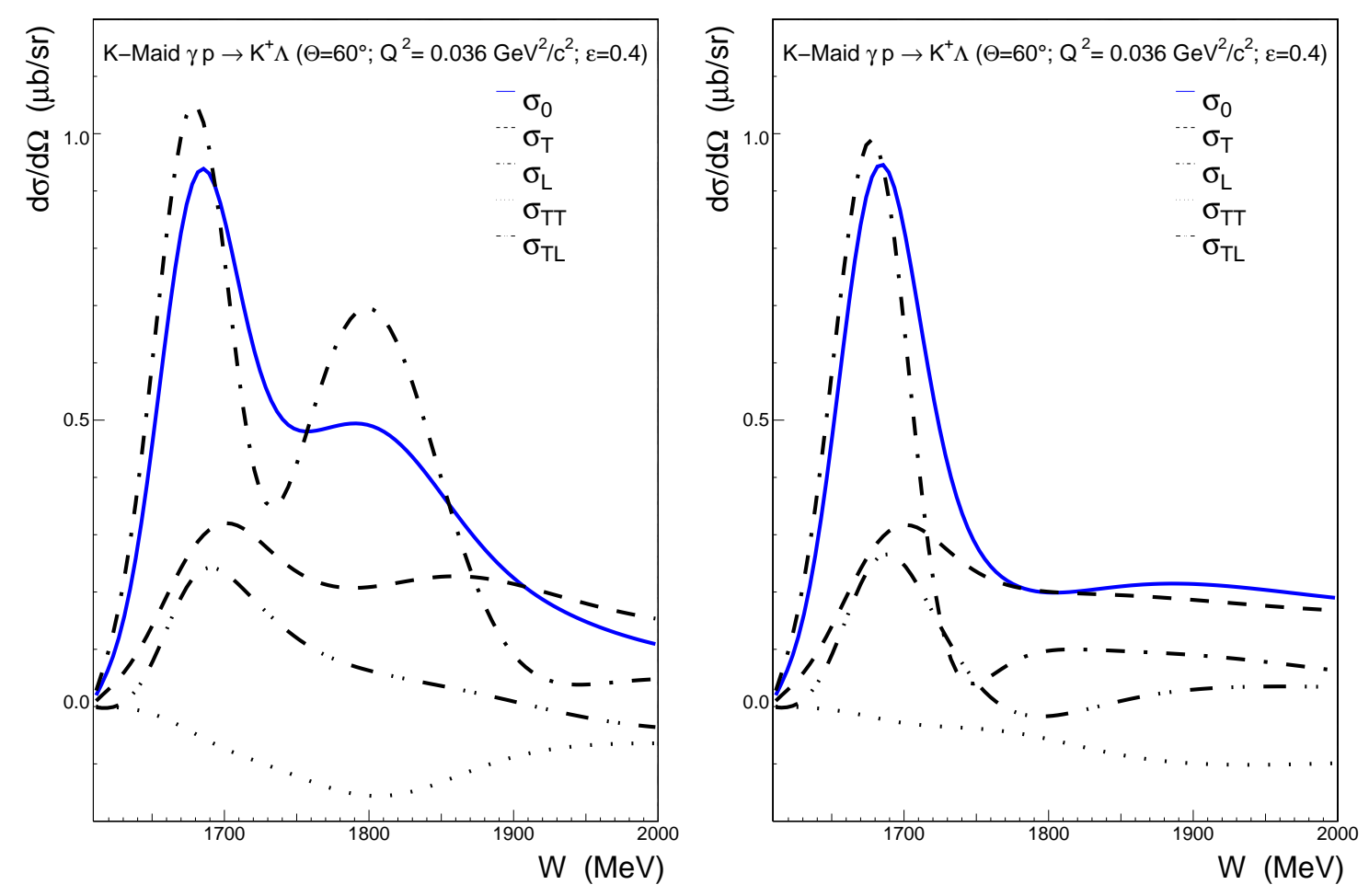

Figure 3: Kaon-Maid cross-section predictions for the $\Lambda$ reaction channel in the kinematics measured at MAMI using the full version (left) and a variant without longitudinal coupling of the $D_{13}(1900)$ resonance (right). The definition of the separated terms of the cross-section is given in the text.

resonance with considerable branching into the strange channel was predicted by the constituent quark model calculations.

Calculations with both models for the kinematics studied with the KAOS spectrometer at MAMI result in relatively large discrepancies in the longitudinal contributions to the cross-section. The full Kaon-Maid model including all couplings and individual dipole form-factors of baryon resonances reveals the following set of cross-sections at the centre of the acceptance in the kinematics probed at MAMI: $\sigma_{T}=235 \mathrm{nb} / \mathrm{sr} ; \sigma_{L}=423 \mathrm{nb} / \mathrm{sr} ; \sigma_{T T}=-121 \mathrm{nb} / \mathrm{sr}, \sigma_{T L}=120 \mathrm{nb} / \mathrm{sr}$. The large longitudinal and the $\sigma_{T T}$, and $\sigma_{T L}$ cross-sections are strongly affected by the longitudinal coupling of the $D_{13}(1900)$ resonance and the appropriate electromagnetic form-factor. With vanishing longitudinal coupling the change in $\sigma_{L}, \sigma_{T T}$, and $\sigma_{T L}$ cross-sections is dramatic. This behaviour of the model is questionable. Fig. 3 shows the predictions using the full version compared to a variant without longitudinal coupling of the $D_{13}(1900)$ resonance in the kinematic region of the measurements at MAMI. The electromagnetic form-factor of the $D_{13}(1900)$ was introduced into Kaon-Maid and fixed to the data taken at Jefferson $\operatorname{Lab}[7,8]$ to produce a sharp peak in $\sigma_{L}$ as a function of $Q^{2}$ at one hadronic energy $(W=1.84 \mathrm{GeV})$.

The model SLA is a simplified version of the full Saclay-Lyon model in which a nucleon resonance with spin-5/2 appears in addition. The extension of the Saclay-Lyon model to the electroproduction does not include too many additional parameters. Predictions of the Saclay-Lyon model are in good agreement with predictions of the discussed variant of the Kaon-Maid model in which 
identical phenomenological electromagnetic form-factors are used and also with those from the original Kaon-Maid version without the longitudinal coupling of the $D_{13}(1900)$ resonance.

These models characterise our present understanding of kaon photo-production reactions at photon energies below $1.5 \mathrm{GeV}$. However, the variations in the model predictions in the resonance region are clearly visible which lead to the conclusion, that our description of the process still lacks considerable insight.

\section{Perspective}

With regard to future experimental work it is obvious that there are interesting topics in elementary kaon electro-production in the region $0<Q^{2}<0.5 \mathrm{GeV} / c^{2}$. The planned operation of the KAOS spectrometer using a pre-target beam chicane provides an experimental set-up that is unique in the world and which will allow unprecedented measurements at forward angles.

Kaon electro-production measurements are only one of the many perspectives from which strangeness physics should be viewed, with the ultimate goal of providing a more thorough understanding of the composition of nuclear matter and sub-nuclear reaction dynamics.

\section{Acknowledgements}

I am thankful to Terry Mart from the University of Indonesia and Petr Bydžovský from the Nuclear Physics Institute in Řež near Prague for sending me their codes for the calculation of kaon photoelectro-production cross-sections and who assisted me in the interpretation of the results.

\section{References}

[1] M. Q. Tran et al., Measurement of $\gamma p \rightarrow K^{+} \Lambda$ and $\gamma p \rightarrow K^{+} \Sigma^{0}$ at photon energies up to $2 \mathrm{GeV}$, Phys. Lett. B 445 (1998) 20-26.

[2] K. H. Glander et al., Measurement of $\gamma p \rightarrow K^{+} \Lambda$ and $\gamma p \rightarrow K^{+} \Sigma^{0}$ at photon energies up to $2.6 \mathrm{GeV}$, Eur. Phys. J. A 19 (2004) 251-273.

[3] M. Iodice, Kaon electro-production on protons at JLab in Hall A, Eur. Phys. J. A 17 (2003) 345-348.

[4] D. S. Carman et al., First measurement of transferred polarization in the exclusive $\vec{e} p \rightarrow e^{\prime} K^{+} \vec{\Lambda}$ reaction, Phys. Rev. Lett. 90 (2003) 131804.

[5] P. Ambrozewicz et al., Separated structure functions for the exclusive electroproduction of $\mathrm{K}^{+} \Lambda$ and $K^{+} \Sigma^{0}$ final states, Phys. Rev. C 75 (2007) 045203.

[6] M. E. McCracken et al., Differential cross section and recoil polarization measurements for the $\gamma p \rightarrow K^{+} \Lambda$ reaction using CLAS at Jefferson Lab, Phys. Rev. C 81 (2010) 025201.

[7] G. Niculescu et al., Longitudinal and transverse cross sections in the ${ }^{1} H\left(e, e^{\prime} K^{+}\right) \Lambda$ reaction, Phys. Rev. Lett. 81 (1998) 1805-1808.

[8] R. M. Mohring et al., Separation of the longitudinal and transverse cross sections in the ${ }^{1} H\left(e, e^{\prime} K^{+}\right) \Lambda$ and ${ }^{1} H\left(e, e^{\prime} K^{+}\right) \Sigma^{0}$ reactions, Phys. Rev. C 67 (2003) 0552025.

[9] T. Mart, Progress and issues in the electromagnetic production of kaon on the nucleon, arXiv:0904.3601v1 [nucl-th] (2009). 
[10] R. A. Adelseck and B. Saghai, Kaon photoproduction: Data consistency, coupling constants, and polarization observables, Phys. Rev. C 42 (1990) 108-127.

[11] R. A. Williams, C.-R. Ji and S. R. Cotanch, Hyperon electroproduction in a crossing and duality constrained model, Phys. Rev. C 46 (1992) 1617-1635.

[12] C. Bennhold, H. Haberzettl and T. Mart, A new resonance in $K^{+} \Lambda$ electroproduction: The $D_{13}(1895)$ and its electromagnetic form factors, arXiv:nucl-th/9909022 (1999).

[13] T. Feuster and U. Mosel, Photon and meson induced reactions on the nucleon, Phys. Rev. C 59 (1999) 460-491.

[14] R. Shyam, O. Scholten and H. Lenske, Associated photoproduction of $K^{+}$mesons off protons within a coupled-channels K-matrix approach, Phys. Rev. C 81 (2010) 015204.

[15] E. Amaldi, S. Fubini and G. Furlan, Pion electroproduction. Electroproduction at low-energy and hadron form-factors, Springer Tracts in Mod. Phys. 83 (1979).

[16] S. Janssen, J. Ryckebusch and T. Van Cauteren, Constraints on background contributions from $\mathrm{K}^{+} \Lambda$ electroproduction, Phys. Rev. C 67 (2003) 052201.

[17] T. Mizutani, C. Fayard, G.-H. Lamot and B. Saghai, Off-shell effects in the electromagnetic production of strangeness, Phys. Rev. C 58 (1998) 75-90. 